\title{
A non-iterative approach for the modelling of quasi-brittle materials
}

\author{
R. Graça-e-Costa $\cdot$ J. Alfaiate • \\ D. Dias-da-Costa $\cdot$ L. J. Sluys
}

Received: 30 December 2011 / Accepted: 11 September 2012 / Published online: 16 October 2012

(C) Springer Science+Business Media Dordrecht 2012

\begin{abstract}
Due to the softening behaviour of quasibrittle materials, in particular the localisation of initially diffused cracking, convergence problems are often found using an iterative procedure, such as the Newton-Raphson method. This is why a new noniterative procedure is adopted in this paper, which is inspired by the sequentially linear approach (SLA)
\end{abstract}

R. Graça-e-Costa · J. Alfaiate

ICIST, Av. Rovisco Pais, 1049-001 Lisbon, Portugal

D. Dias-da-Costa

INESC Coimbra, Rua Antero de Quental 199,

3000-033 Coimbra, Portugal

R. Graça-e-Costa ( $\square)$

Department of Civil Engineering, Instituto Superior de Engenharia, Universidade do Algarve, Campus da Penha, 8000-117 Faro, Portugal

e-mail: rcosta@ualg.pt

\section{J. Alfaiate}

Department of Civil Engineering and Architecture, Instituto Superior Técnico, Technical University of Lisbon, Av. Rovisco Pais 1, 1049-001 Lisbon, Portugal

e-mail: alfaiate@civil.ist.utl.pt

D. Dias-da-Costa

Department of Civil Engineering, University of Coimbra, Rua Luís Reis Santos, 3030-788 Coimbra, Portugal e-mail: dias-da-costa@dec.uc.pt

\section{J. Sluys}

Department of Civil Engineering and Geosciences, Delft University of Technology, Delft, The Netherlands e-mail: L.J.Sluys@tudelft.nl
(Rots et al. in Eng Fract Mech 75(3-4):590-614, 2008). However, several important differences between the present approach and the SLA are presented. In the present model, multi-linear material laws are adopted such that non-linearities occur only due to changes in loading/unloading states. An incremental solution is obtained until non-convergence occurs, upon which a secant approach is used in a corresponding step. The update of the stiffness in the secant approach is based on information obtained from the previous incremental solution. This method is applied to: (i) softening materials, within the scope of the discrete crack approach, and to (ii) hardening materials. As a consequence, conversely to the smeared crack approach adopted in the $S L A$, no mesh size sensitivity problems are obtained and there is no need to adjust material parameters. Several numerical examples are shown in order to illustrate the proposed formulation.

Keywords NIEM - Fracture - Non-iterative procedure $\cdot$ Sequentially linear analysis

\section{Introduction}

The use of iterative procedures for the nonlinear analysis of structures, namely the Newton-Raphson method and the Arc Length algorithm, often lead to convergence difficulties. The numerical simulation of the softening behaviour of quasi-brittle materials, such as concrete and masonry, associated to the localisation of microcracking into stress-free cracks, is still 
challenging. Moreover, apart from fracture, reinforced concrete exhibits several other nonlinearities such as concrete crushing, the bond-slip between concrete and reinforcement and plastic behaviour of steel reinforcement, which imply the use of many iterations and a large number of steps, thus a considerable amount of time.

In this paper, two new non-iterative procedures are presented for the numerical analysis of concrete structures. These are incremental methods which, whenever critical bifurcation points are found, make use, on that particular load step, of a total/secant approach inspired by the Sequentially Linear Approach from Rots and co-workers (Rots 2001). In this manner, it is possible to analyse reinforced concrete structures until failure, keeping the advantages of an incremental procedure, in which material memory is kept, and the efficiency of the total analysis to overcome convergence difficulties. The paper is organised as follows: in the next section a small literature review on non-iterative methods is first presented. Next, the adopted non-iterative procedures are introduced. The numerical examples are presented in Sect. 3.

\section{Non-iterative procedures}

It is generally assumed that the use of iterative procedures (Crisfield 1984), such as the Newton-Raphson method with or without the coupling to an arc-length method, are unwieldy and, in some cases, unable to provide a converged solution. As a consequence, other methods have been developed which: (i) either completely avoid iterations (Yamada et al. 1968; Rots et al. 2008; Xing and Zhang 2009) or (ii) minimise their use (Oliver et al. 2008).

The sequentially linear approach (SLA) was developed by Rots (2001) and is an event-by-event strategy. It is a secant stiffness based method that does not require iterations and that has been successfully applied within the scope of the smeared crack approach. In the work presented by Graça-e-Costa (2005), Graça-e-Costa and Alfaiate (2006), this procedure was extended to the discrete crack approach, using strong embedded discontinuities to model concrete beams both internally and externally reinforced. These beams were first loaded until a certain level of prescribed damage was attained, repaired and strengthened with externally bonded steel plates glued with epoxy, and reloaded until complete failure of the specimen occurred.

In the work previously referred to, good numerical results were obtained concerning both load displacement curves and crack prediction. In the SLA, the update of the secant stiffness gives rise to a "sawtooth" stress-strain envelope, leading to deviations of the dissipated energy with respect to a standard smeared crack approach. Furthermore, with this first non-regularised version of the SLA, the consistency condition is often violated since an increase of damage may occur on material points which no longer stay on the limit surface. In order to obtain objective results with respect to the mesh, as well as to overcome the lack of consistency, the material law was converted into a "saw-tooth" stress-strain law, which provides the correct energy dissipation (Rots et al. 2008) (see Fig. 1).

This method has been successfully applied to masonry structures (Rots 2001; Rots et al. 2008), reinforced concrete beams (Graça-e-Costa 2005; Graça-eCosta and Alfaiate 2006), composite beams (Billington 2009), concrete beams exhibiting shear failure (Slobbe et al. 2012), structures composed by extremely brittle materials, such as glass (Invernizzi et al. 2011), and concrete in general, providing good results in problems which are difficult to solve due to non-convergence problems.
Fig. 1 Regularised saw-tooth softening envelopes in the SLA (Rots et al. 2008). a Linear, b exponential (a)

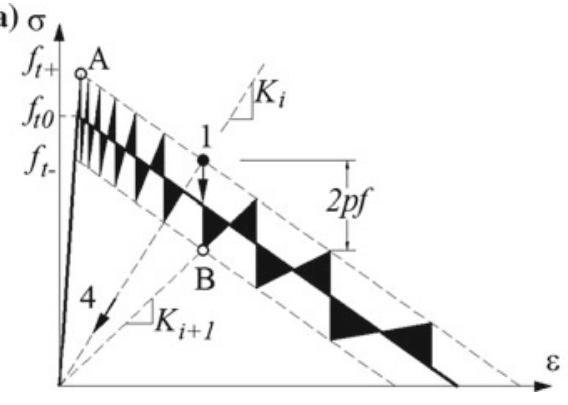

(b)

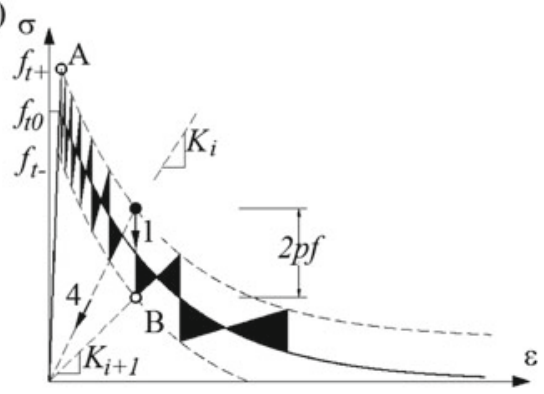


In the present paper, two innovative approaches are proposed. Conversely to the SLA, multi-linear material laws are adopted such that all material non-linearities are exclusively due to changes in loading/unloading states.

First, a non-iterative incremental solution is obtained until non-convergence occurs, upon which a total approach is used in the corresponding step only. The stiffness update in the total approach is based according to: (i) the $S L A$, or (ii) the information obtained from the previous incremental solution.

The first method is called the automatic method and the second method is called the non-iterative energy based method (NIEM). The two methods are applied to both: (i) softening materials, within the scope of the discrete crack approach, and to (ii) hardening materials. As a consequence, and conversely to the smeared approach adopted in the SLA, no mesh size sensitivity is obtained and there is no need to adjust the material parameters and/or the material law.

\subsection{Solution control}

In the incremental approach, it is necessary to control the solution on a bifurcation path as illustrated in Fig. 2 for a uniaxial traction-jump law. Apart from the standard load and displacement controls, several methods are available such as the enforcement of a monotonic opening of a notch, which has also been experimentally used. In the present study and whenever no experimental evidence is known, the method used to choose the load path is based on the energy release control method (Gutiérrez 2004; Verhoosel et al. 2009).

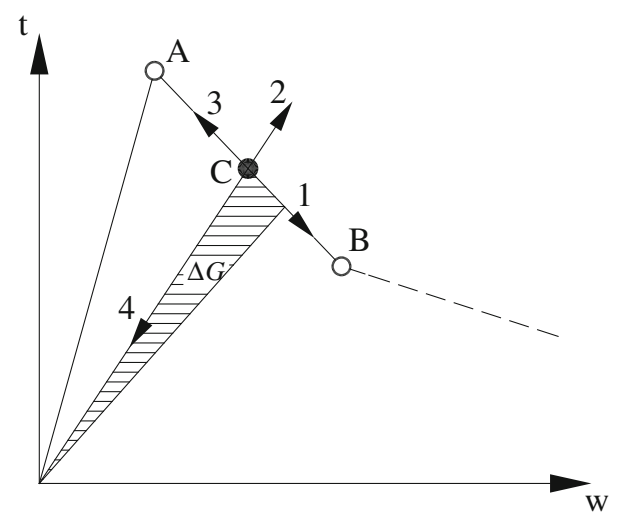

Fig. 2 Possible traction-jump paths
This method was proposed in order to create a constraint in the arc-length algorithm: for a body under a load increment, the corresponding stress-strain evolution of the material point follows the path which gives rise to the largest energy release. Upon using an incremental approach, the second order variation of the energy release in the whole structure (bulk and discontinuities), $\Delta G$, is given by;

$$
\Delta G=\int_{\Omega} \Delta \boldsymbol{\varepsilon}^{T} \Delta \boldsymbol{\sigma} d \Omega+\int_{\Gamma_{d}} \Delta \mathbf{w}^{T} \Delta \mathbf{t} d \Gamma_{d},
$$

where $\Omega$ is the bulk and $\Gamma_{d}$ stands for all discontinuities, $\boldsymbol{\varepsilon}$ and $\sigma$ are the strains and the stresses in the bulk, respectively, and $\mathbf{w}$ and $\mathbf{t}$ are the jump displacements and the tractions, respectively, at the discontinuities.

In the new procedures, the global second order variation of energy consumption in the current step, obtained at structural level, is designated: (i) $G_{A}$ for a positive load increment and (ii) $G_{B}$ for a negative load increment. Consider material point $\mathrm{C}$ represented in Fig. 2. Assume that, for a positive load increment, this material point follows path number 1 . In this case, the global variation of energy consumption increases by a certain amount corresponding to the triangle shown in the figure, which is added to variable $G_{A}$. Conversely, a negative load increment would give rise to unloading path number 4, without any additional dissipation of energy. As a consequence, variable $G_{B}$, in which the variation of energy consumption is accumulated for a negative load step, remains unchanged. In other material points the opposite may occur: no energy consumption is accumulated in $G_{A}$ for a positive load step (path number 4), whereas additional variation of energy is added to variable $G_{B}$ for a negative load step, corresponding to path number 1 in Fig. 2. Thus, at structural level, and upon two possible opposite solution directions, one is chosen which leads to the maximum value of $\Delta G$ obtained in the whole structure, i.e., to $\max \left(G_{A}, G_{B}\right)$.

Incrementally, since multilinear constitutive relations are adopted, a critical load factor $\left(\lambda_{\text {crit }}\right)$ is first evaluated in a trial step, in order to reach the nearest material point connecting two linear branches on the envelope (for instance, point B in Fig. 2).

$$
\Delta \mathbf{P}_{\text {true }, j}=\lambda_{\text {crit }} \Delta \mathbf{P}_{\text {trial }, j}
$$

and the material stiffness is updated. 


\subsection{Critical bifurcation points}

When bifurcation points are reached on the material law two possibilities occur: increase of damage or unloading. In Fig. 2, a 1D representation of the constitutive law is shown, in which four paths on the traction-displacement curve are displayed: path 1 corresponds to the increase of damage, paths 2 and 3 are unacceptable since they violate the material law, and path 4 corresponds to secant unloading.

Whenever a material point undergoes unloading, memory is kept until it reloads back to the envelope. When the current state is unloading this point is not critical except for preventing overlapping of crack faces at crack closure; when reloading occurs, the load factor is estimated, similarly to what is done for all points that remain on the envelope.

Generalising, let $\mathbf{w}$ and $\mathrm{d} \mathbf{w}$ be the total and incremental displacement jump vector, respectively, and $\boldsymbol{\varepsilon}$ and $\mathrm{d} \boldsymbol{\varepsilon}$ the total and incremental strain tensor, respectively. Since linearised constitutive laws are adopted, non-convergence can only occur for cases in which the material point lies on the envelope:

(i) the tangent stiffness corresponds to the envelope stiffness and $\mathbf{w} \cdot \mathrm{d} \mathbf{w}<0$ (or $\boldsymbol{\varepsilon} \cdot \mathrm{d} \boldsymbol{\varepsilon}<0$ ), where '. ' is the internal product-case 3 in Fig. 2, or;

(ii) the tangent stiffness corresponds to the unloading stiffness and $\mathbf{w} \cdot \mathrm{d} \mathbf{w}>0($ or $\boldsymbol{\varepsilon} \cdot \mathrm{d} \boldsymbol{\varepsilon}>0)$ —case 2 in Fig. 2. Non-convergence occurs when these two cases happen repeatedly, one after the other.

\subsubsection{Automatic method}

In the automatic method, whenever a critical bifurcation point is reached, after which it is impossible to incrementally determine the effective path, a total method is adopted in which the secant material stiffness is adopted. This secant stiffness is then reduced by a predefined factor as done in the SLA. In Fig. 3, the automatic switch is illustrated in 1D: upon completion of valid step number 3 , in step number 4 a critical bifurcation point is reached. A change to the total formulation is performed and a secant stiffness matrix is defined, according to the previous equilibrium state (point 3 in Fig. 3):

$K_{j}=\frac{t_{j}}{w_{j}}$

where step 3 corresponds to step $j$. In the following step the secant stiffness is reduced, using the standard SLA.

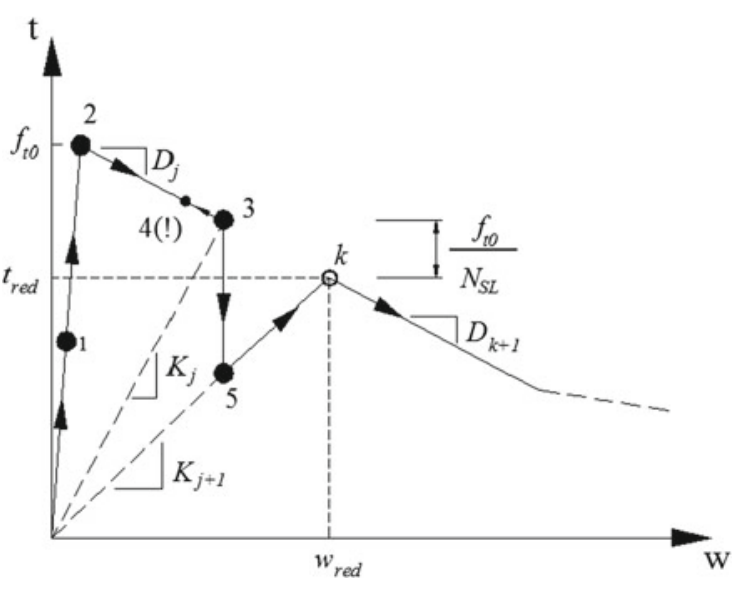

Fig. 3 1D example of the automatic method

Instead of incremental step 4, step $j+1$ is obtained using the secant stiffness which is updated according to a stress reduction:

$t_{\text {red }}=t_{j}-\frac{f_{t 0}}{N_{S L}}$

where $f_{t 0}$ is the tensile strength and $N_{S L}$ is the predefined number of $S L A$ reduction steps. This total step would correspond to step 5 if it was obtained incrementally.

In general, similarly to the $S L A$, several integration points will exhibit a traction decrease due to this approach and will remain below the envelope, since only one will reach the envelope surface under critical load factor $\lambda_{\text {crit }}$ (see Fig. 3). The next step is performed similarly to the $S L A$, in which usually only one of the points will become critical and reaches the envelope on point $k$. All other points will remain on the current secant path. In the following step the incremental approach is recovered using the tangent stiffness matrix D. This algorithm is effective because it tends to follow the real envelope and circumvents critical bifurcation points using a total approach. Moreover, the use of an incremental procedure leads to less computation time than a pure total procedure such as the SLA.

Note that the secant stiffness is always adopted in the total approach, which has a direct correspondence to damage behaviour under softening. In elastoplastic materials it is also possible to enforce the correct unloading path using the same total approach; in this case, the secant stiffness is only adopted to reach new equilibrium positions on either: (i) the loading surface or (ii) the unloading surface. 
Fig. 4 Flowchart for the automatic method. (*) All integration points follow valid paths in global loading situation, adopt positive load increment. $\left(^{\dagger}\right)$ All integration points follow valid paths in global unloading situation, adopt negative load increment

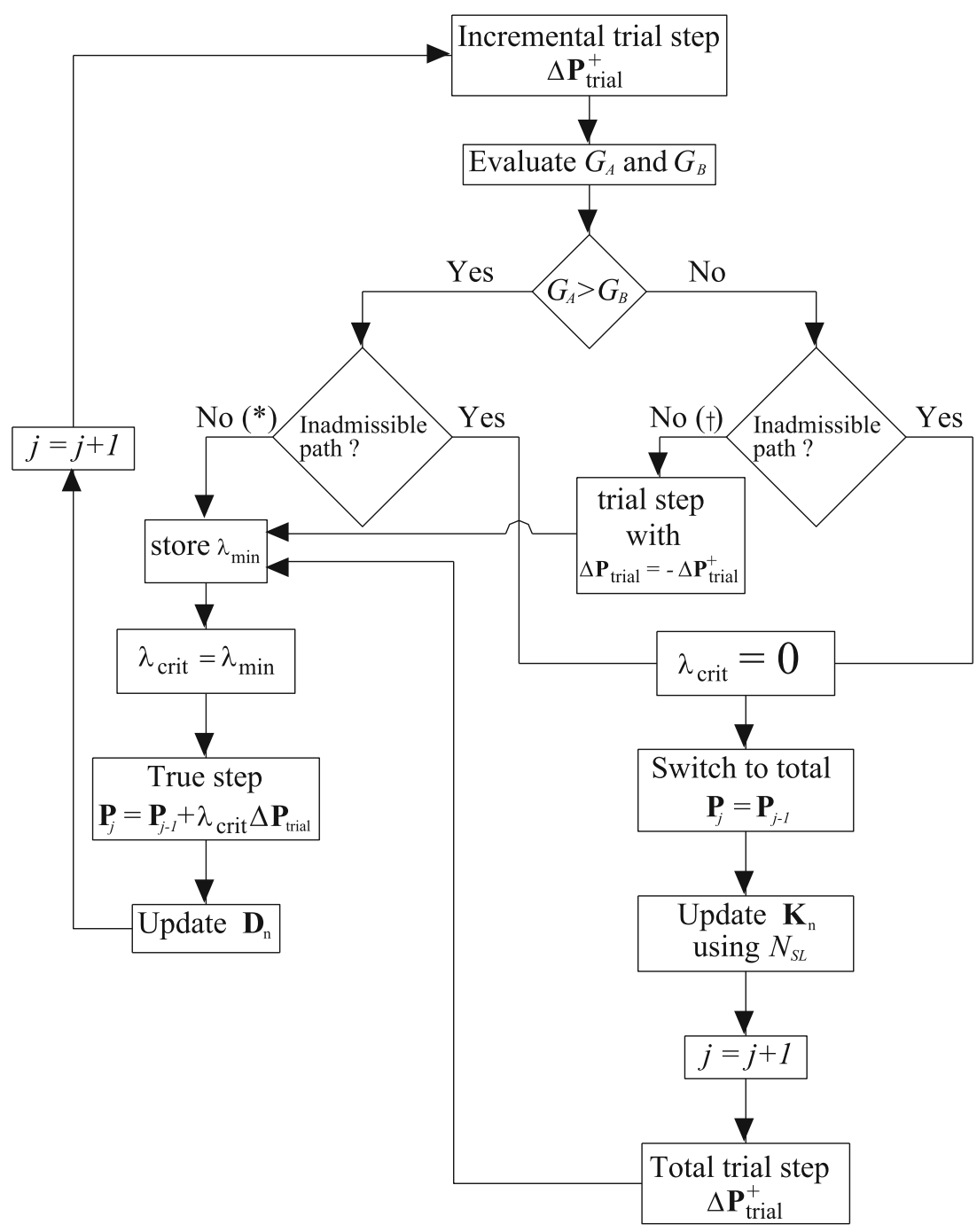

For a more comprehensive explanation, a flowchart of the procedure is presented in Fig. 4. First, a positive load increment $\Delta \mathbf{P}_{\text {trial }}^{+}$is imposed; next, after evaluation of the dissipated energies $G_{A}$ and $G_{B}$, either $G_{A}>$ $G_{B}$ and the incremental load sign is kept, or $G_{B}>G_{A}$ and the sign of the load increment is reversed. If at least one inadmissible path is obtained, then impose a null step and switch to total; otherwise, apply critical load step factor $\lambda_{\text {min }}$, update the material law and proceed to the next step.

\subsubsection{Non-iterative energy based method}

The automatic method is an effective tool for numerical non-linear analysis. Furthermore, it overcomes the need to modify both material parameters and material law in order to approximate the correct fracture energy consumption. However, the stepwise decrease of the secant stiffness must be defined a priori, without a clear meaning. In order to avoid this situation a new method is introduced, which allows for switching between the incremental and the total approach without imposing a predefined number of reductions of the secant stiffness.

Assume first that a valid incremental step is performed. In this case, all material points lying on the envelope will evolve either along the envelope or unload. Thus, after the true step, it is possible to identify the new updated secant stiffness for each point. This is the main idea behind the NIEM: when critical bifurcation points are found, switch to the total approach 


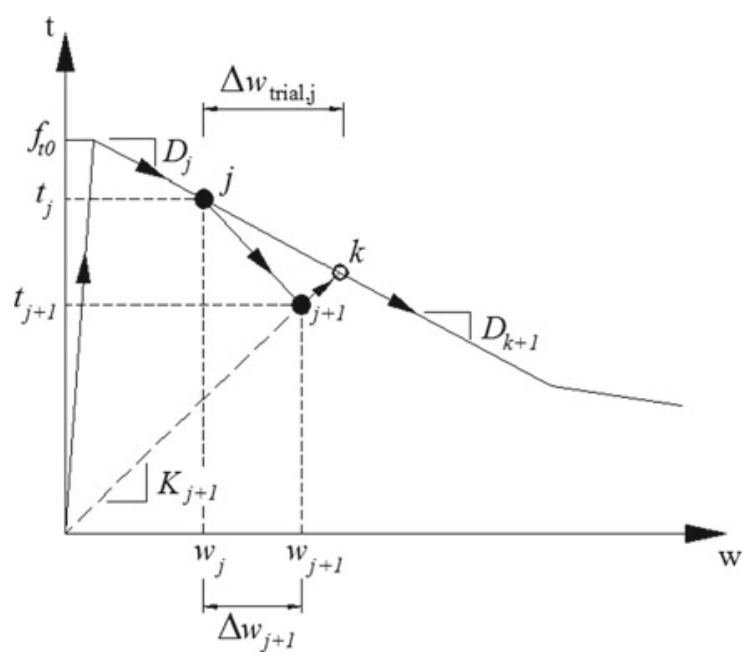

Fig. 5 1D example of the NIEM

and let all material points evolve on the secant stiffness which would emerge from a valid (virtual) incremental step, keeping fixed the secant stiffness of the critical bifurcations points. In this way, the total solution is expected to better approximate the consistency condition. The illustration of this procedure from step $j$ to $j+1$ is shown in Fig. 5 for a non-critical point. Point $k$ and the corresponding secant stiffness $K_{j+1}$ is estimated according to the trial step $j$ :

$K_{j+1}=\frac{t_{j+1}}{w_{j}+\Delta w_{\text {trial }, j}}$

Thus, in step $j$ a null step is enforced and only evaluation of the new secant stiffness is performed, which will be used in step $j+1$ using a total approach. The following steps are incremental, following the current secant until point $k$ is reached, after which the path on the material envelope is followed again.

An explanative flowchart for this method, is presented in Fig. 6. As will be demonstrated later, this method leads to smaller deviations from the material law than the previous automatic method since it is based on model prediction instead of a predefined update.

\section{Structural examples}

In this section, numerical examples are used to illustrate the capabilities of the proposed procedures: (i) a plain concrete double-cantilever test with severe snap-backs due to high brittleness; (ii) a single edge notched beam; (iii) a three point bending beam; (iv) a masonry panel; (v) a concrete dam and (vi) a reinforced concrete beam under monotonic loading. In the first case, it is shown that a proper choice of solution control is essential to capture all bifurcation points, whereas in the second and third, it is demonstrated the viability of adopting linearised softening diagrams. In the latter cases, the new methodology is applied to structures with critical bifurcation points, in which localisation of initially severe distributed cracking is difficult to capture with conventional iterative methods.

\subsection{Double cantilever beam}

In this section a double cantilever beam test is presented (see Fig. 7), using an extremely small value of the fracture energy $\left(G_{F}=0.001 \mathrm{~N} / \mathrm{mm}\right)$. The goal of this test is to demonstrate that the energy control method described in Sect. 2.1 seems to be the only efficient solution control criterion in the presence of sharp snap-backs. The test is simple, giving rise to a vertical mode-I crack at the symmetry line. The crack is modelled by 8 interface elements, with a tensile strength value $f_{t 0}=3.0 \mathrm{~N} / \mathrm{mm}^{2}$. A bi-linear softening envelope was used.

The use of small fracture energy causes a fast energy release, as observed in brittle materials such as glass. Each interface first opens and afterwards closes, after dissipating energy, before the next one starts developing, upon which it reopens. It is clear that in this case load or displacement control procedures cannot be adopted. Moreover, even the monotonic opening of the crack tip (or any other point along the crack) does not occur.

In Fig. 8 the load-CMOD curves are presented both with:

(i) the incremental approach (24 steps), in which the energy control method introduced in 2.1 is adopted; and;

(ii) the total approach (80 steps).

In the first case, in which the loading is clearly nonmonotonic, no critical bifurcation points are found, whereas in the latter case, which also proves to be adequate, small differences can be found due to fracture energy underestimation, inherent to this approach since no correction of the material parameters is performed. 
Fig. 6 Flowchart for the NIEM. (*) All integration points follow valid paths in global loading situation, adopt positive load valid paths in global unloading situation, adopt negative load increment increment. $\left(^{\dagger}\right)$ All integration points follow

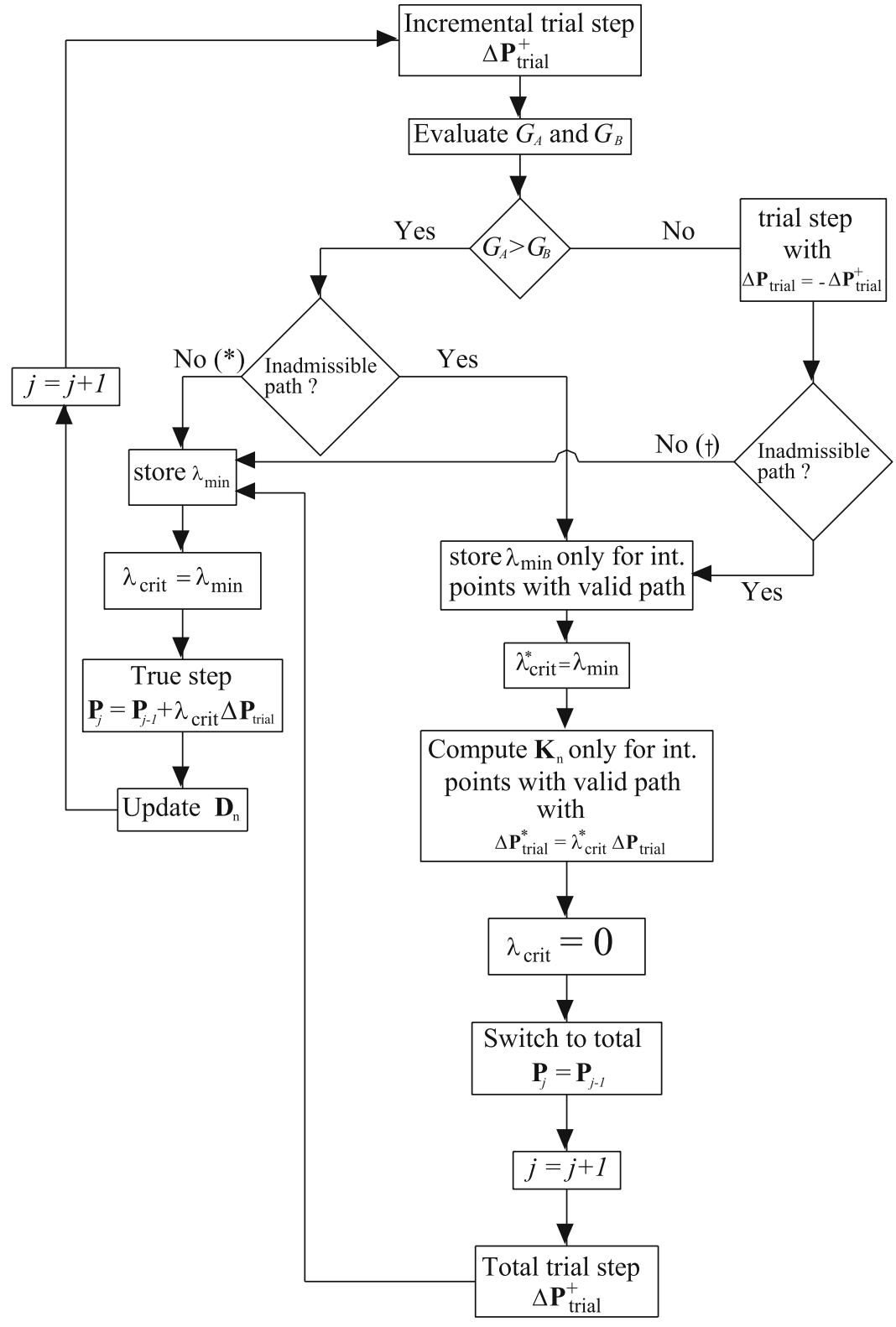

Fig. 7 Double cantilever beam: structural scheme and finite element mesh $(1 \mathrm{~mm}$ width, dimensions in $\mathrm{mm}$ )
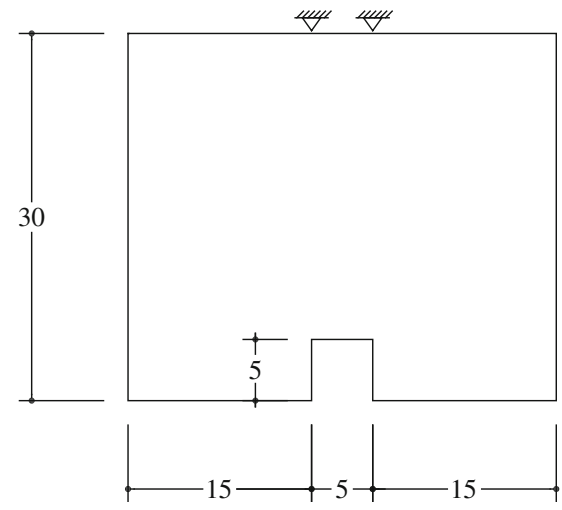

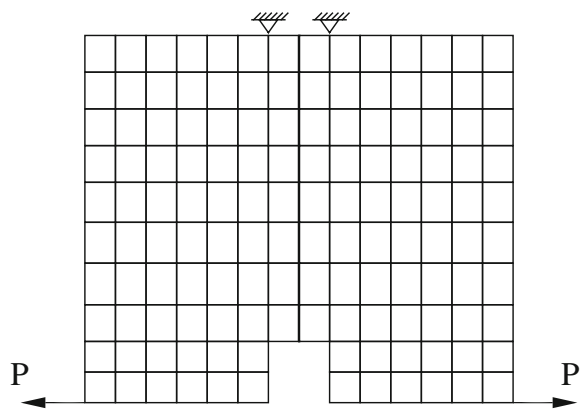




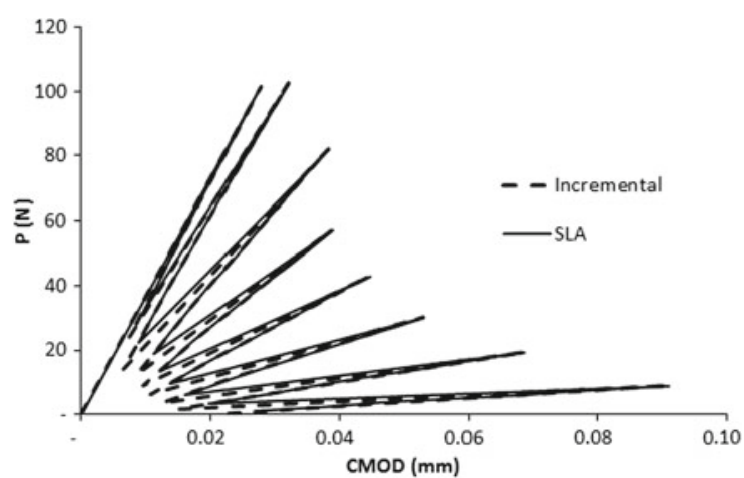

Fig. 8 Double-cantilever beam: load-CMOD curves

\subsection{Single edge notched beam}

The single edged notched beam model is a common benchmark test in numerical modelling and it is based on the experimental work of Schlangen (1993). It has been used for the assessment of the role of mode-II fracture even though the author concluded that the fracture mechanism is mode-I dominated. The latter occurs even if the global mechanism is a combination of modeI and mode-II fracture since cracking occurs perpendicularly to the maximum tensile stress in the beam, associated with parallel displacements between the crack faces. This way, mode-I behaviour was adopted with isotropic softening (this aspect is commentated in Alfaiate et al. 2002)

This test consists of a beam with two supports creating a cantilever and a double supported span with a notch from which the major crack emerges.

The beam is composed of normal weight concrete with $8 \mathrm{~mm}$ maximum aggregate size and has global measures of $400 \times 100 \times 100 \mathrm{~mm}^{3}$ and a $5 \times 20 \times$ $100 \mathrm{~mm}^{3}$ notch located at the top, as shown in Fig. 9.

The material parameters are: Young's modulus $E=35,000 \mathrm{~N} / \mathrm{mm}^{2}$; Poisson's coefficient $\nu=0.15$; tensile strength $f_{t 0}=3.0 \mathrm{~N} / \mathrm{mm}^{2}$ and fracture energy

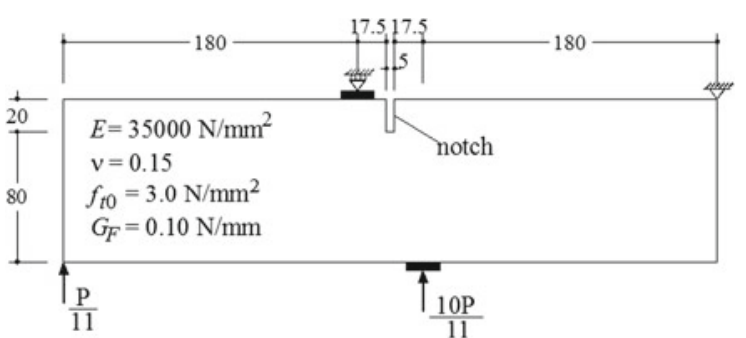

Fig. 9 Single-edge notched beam: structural scheme, load and boundary conditions (100 $\mathrm{mm}$ width, dimensions in $\mathrm{mm}$ )

$G_{F}=0.1 \mathrm{~N} / \mathrm{mm}$. A bi-linear softening envelope was used with a transition point between the two stiffness branches defined by $w_{1}=0.0267 \mathrm{~mm}$ and $f_{t 1}=$ $1.0 \mathrm{~N} / \mathrm{mm}^{2}$. The limit jump value is $w_{\text {ult }}=0.12 \mathrm{~mm}$.

The load control procedure consists in monotonically increasing the relative sliding displacement of the notch (crack mouth slide displacement-CMSD). This ensures that no critical bifurcation points are found, allowing, once again, the use of a purely incremental analysis. In this way it is possible to validate the use of linearised curves associated to the incremental approach.

The finite element mesh is presented in Fig. 10. Two analysis were made using an incremental approach and a total approach based on the SLA with modified material properties as defined in Rots et al. (2008), here adapted to a bi-linear envelope.

The resulting load-CMSD curve from both formulations is close to the experimental result, presenting a similar peak load and pre and post-peak stiffness (Fig. 11). As expected, the incremental curve is smooth although only 58 steps are needed to complete the analysis. In the case of the SLA, the curve is not smooth, but follows the incremental solution, with points above and below, corresponding to the fracture energy compensation method used on the SLA. In this case, 216 steps are necessary.
Fig. 10 Single-edge notched beam: finite element mesh

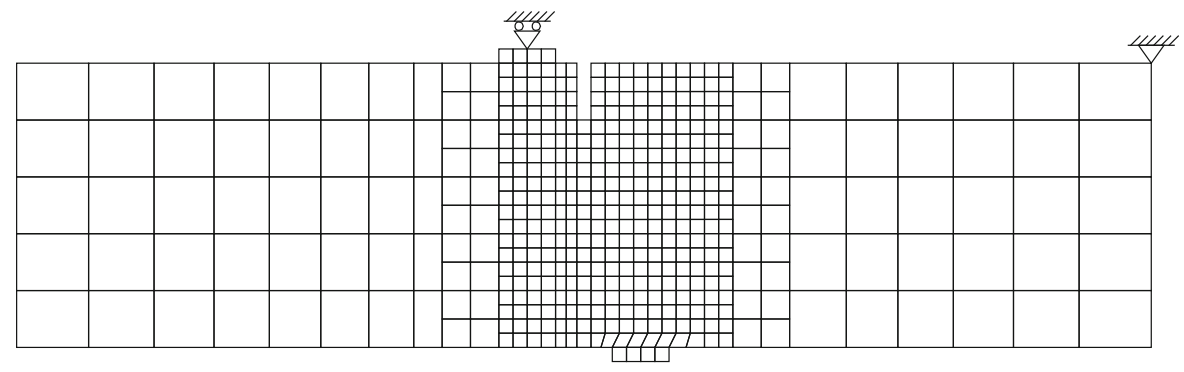




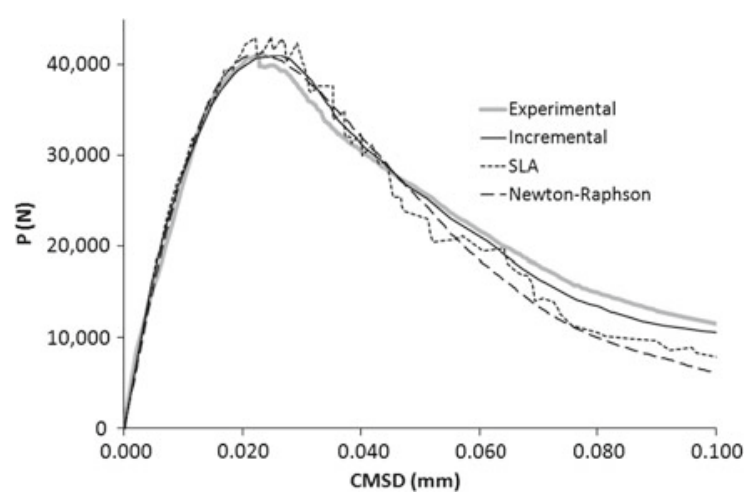

Fig. 11 Single-edge notched beam: load-CMSD curves

Both deformed shape and major crack are shown in Fig. 12. In order to evaluate the accuracy of both approaches, the obtained traction-jump envelope curves at the notch are plotted in Figs. 13 and 14. In the incremental case, the original envelope is perfectly captured and the dots represent each step. For simplicity, only the steps where the adopted integration point was critical (change in tangent stiffness) are represented.

The saw-tooth shape of the SLA is a consequence of the procedure, and the use of mesh regularisation proves to overcome mesh dependencies. However the obtained value of fracture energy is overestimated $\left(G_{F}=0.1098 \mathrm{~N} / \mathrm{mm}\right)$ which corresponds to an increase of $9.8 \%$.

\subsection{Galvez's notched beam}

Another test for which results can be obtained incrementally is the Galvez's notched beam (Galvez et al. 1998), which consisted in the experimental characterisation of the mixed mode fracture for notched beams. The model is a three point bending notched concrete beam with a cantilever span that has a $64^{\circ}$ linear path prescribed crack. The beam has the mechanical properties shown in Fig. 15.

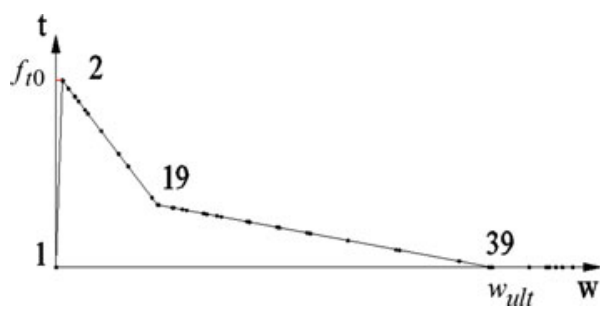

Fig. 13 Single-edge notched beam: traction-jump envelope (MPa-mm) at the major crack tip using the incremental approach

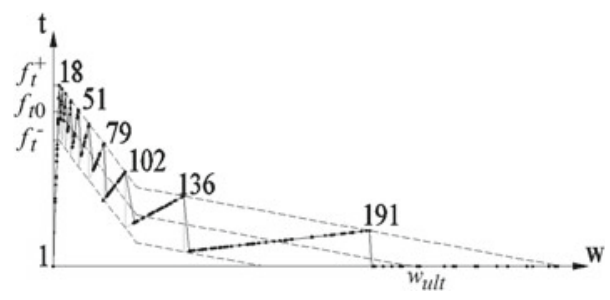

Fig. 14 Single-edge notched beam: saw-tooth traction-jump envelope (MPa-mm) at the major crack tip using the $S L A$

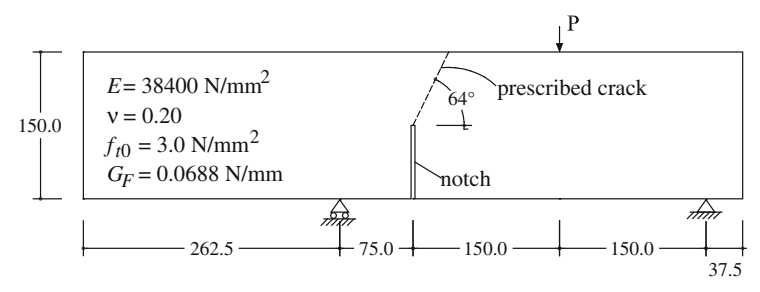

Fig. 15 Galvez's beam: structural scheme, load and boundary conditions (50 $\mathrm{mm}$ width, dimensions in $\mathrm{mm}$ )

The finite element model is an unstructured mesh, composed of 1,217 elements, with a linear behaviour law for concrete under compression, since failure is governed by mode-I cracking. The adopted mode I softening law is bi-linear $\left(w_{1}=0.0183 \mathrm{~mm}\right.$ and $f_{t 1}$ $\left.=1.0 \mathrm{~N} / \mathrm{mm}^{2}, w_{\text {ult }}=0.0826 \mathrm{~mm}\right)$, whereas the shear stiffness drops to zero when softening occurs.

The solution control adopted consists in monotonically increasing the crack mouth opening displacement
Fig. 12 Single-edge notched beam: deformed shape at CMSD $=0.1 \mathrm{~mm}$ (displacements amplified 100 times)

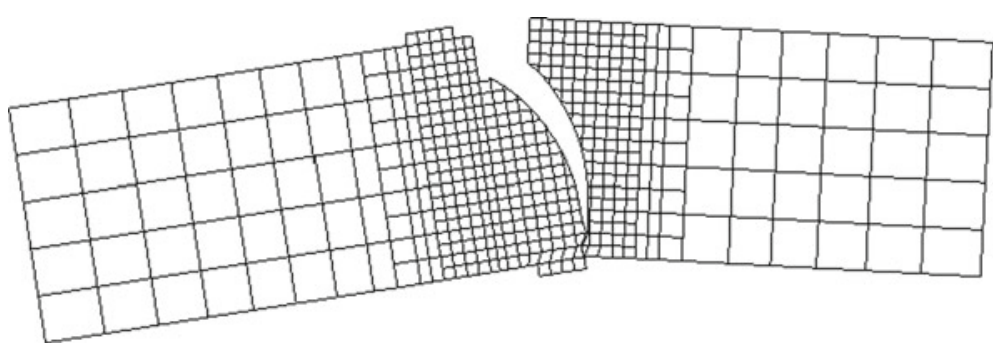




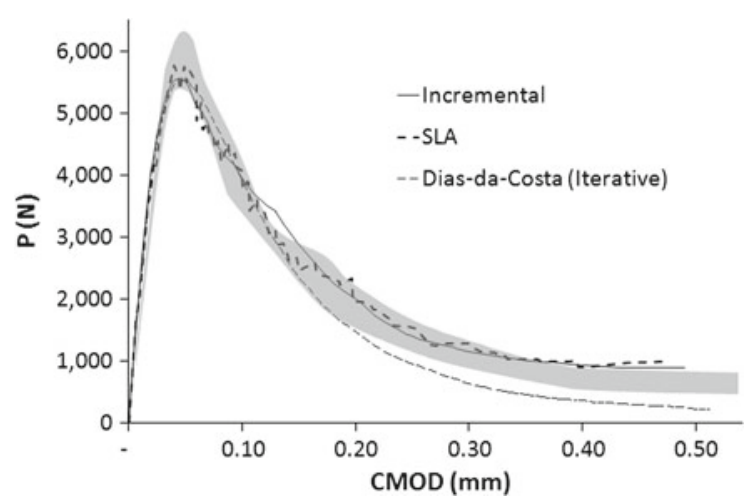

Fig. 16 Galvez's beam: load-CMOD curves

(CMOD). The two approaches used lead to good results when comparing the load-CMOD curves with the experimental results obtained by Galvez (Fig. 16). In both cases, the peak load and the softening branch lay inside the experimental envelope. An additional curve is represented in Fig. 16 which was obtained by Diasda-Costa et al. (2009). In this case, an iterative procedure was adopted, using the arc-length method, and cracking was simulated using the discrete strong discontinuity approach (DSDA).

The results using the incremental approach are obtained with 78 steps and the total approach with 241 steps). In Fig. 17, the deformed mesh obtained with the incremental approach is presented. A similar result was obtained with the SLA.

It is not possible to make a direct comparison among the non-iterative solutions and the iterative solution, since the non-iterative solutions are obtained after running the program once, whereas each iterative solution needs several runs of the program, with calibration of the step sizes, testing of different arc length constraints and so forth, often taking an enormous amount of time before an optimised converged solution can be obtained. Nevertheless, comparison between this optimised iterative solution and the non-iterative solutions is the following: the iterative solution took $117 \mathrm{~s}$ whereas the incremental solution took 39 s and the $S L A$ solution took $106 \mathrm{~s}$.

\subsection{Historical masonry wall test}

A test campaign to evaluate the seismic risk of old masonry buildings is currently being developed at the Instituto Superior Técnico in Lisbon. The Severes project has several objectives, but for the scope of the present paper, a single type of test will be analysed which consists of a diagonal load solicitation on masonry walls (Fig. 18). Properties, geometry and loading path can be found in Milosevic et al. (2010) and Gago et al. (2011). These walls were built in accordance to the standard procedures of the eighteenth century, using roughly cut stone (limestone) and hydraulic or air lime mortar. The cited report presents two different tests, one with hydraulic mortar and one with air lime mortar. On the present numerical analysis, only the tests with hydraulic mortar are simulated.

The material is homogenised using equivalent properties obtained from a parametric study which led to the adopted Young's modulus $E=1,700 \mathrm{MPa}$. To model compression in the bulk a multi-linear envelope was used, with elastic behaviour until 1.85 MPa stress and with a limit stress of $4.00 \mathrm{MPa}$, followed by an yielding horizontal branch, the used Poisson's coefficient value is 0.20 and the tensile strength $f_{t 0}=0.25 \mathrm{~N} / \mathrm{mm}^{2}$. The following parameters were also estimated: cohesion for mode II, $c_{0}=0.05 \mathrm{MPa}$; normal stiffness for closed discontinuities, $k_{n}=10^{10} \mathrm{~N} / \mathrm{mm}^{3}$; Fracture energy $G_{F}=0.10 \mathrm{~N} / \mathrm{mm}$. A bi-linear softening law was adopted $\left(w_{1}=0.32 \mathrm{~mm}\right.$ and $f_{t 1}=0.083 \mathrm{~N} / \mathrm{mm}^{2}$, $\left.w_{u l t}=1.44 \mathrm{~mm}\right)$.
Fig. 17 Galvez's beam: deformed shape at CMOD $=$ $0.5 \mathrm{~mm}$ (displacements amplified 100 times)

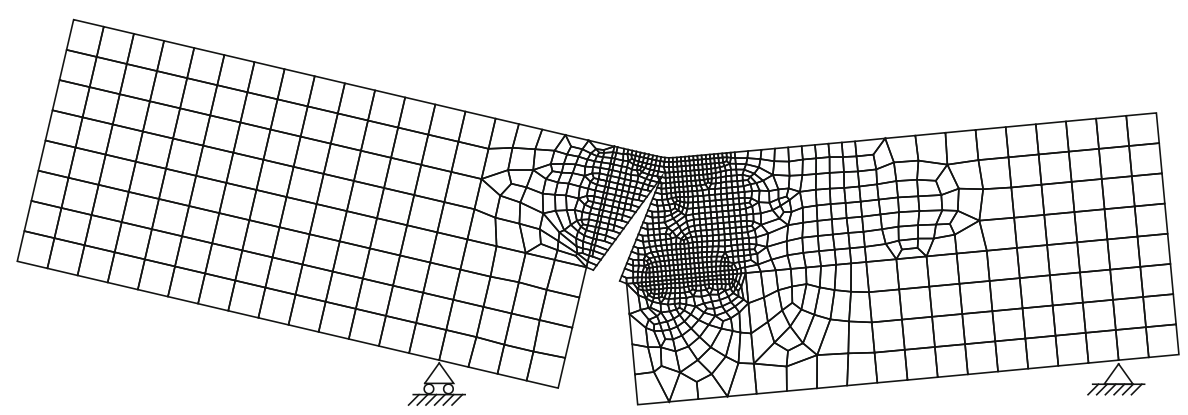


Fig. 18 Severes diagonal test: a illustration of the test. b Finite element mesh. (700 mm width, dimensions in $\mathrm{mm}$ )

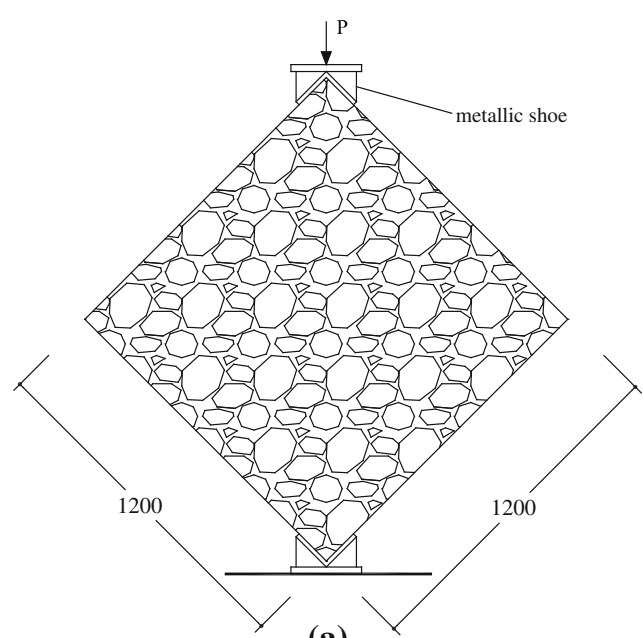

(a)

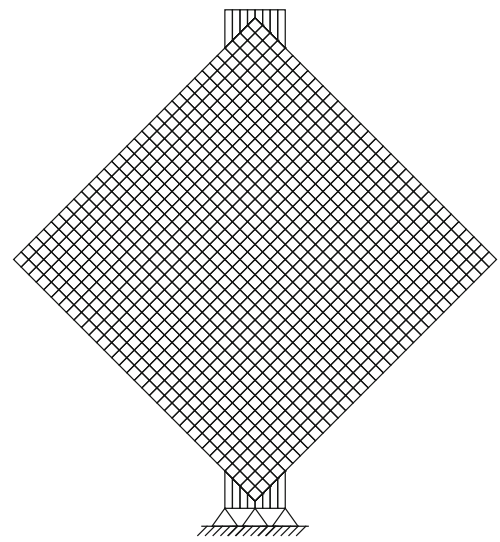

(b)

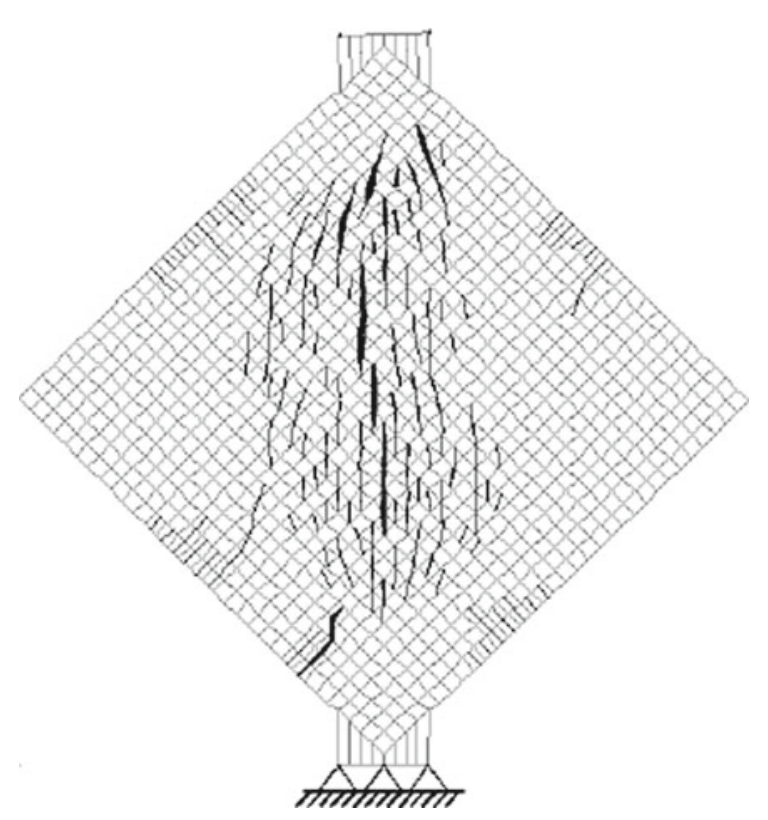

Fig. 19 Severes diagonal test: deformed finite element mesh and crack pattern using the automatic method

In this highly non-linear model, several cracks open at the same time as other cracks unload, giving rise to many critical bifurcation points. Thus, this is an excellent example to test the proposed methods.

Two models are computed. In the first one non-prescribed embedded discontinuities are used (see results in Figs. 19 and 20). In this case the panel tends to exhibit a natural lateral expansion that leads to vertical crack formation. The load-displacement curves are close to the experimental curve, with 650 steps for the total

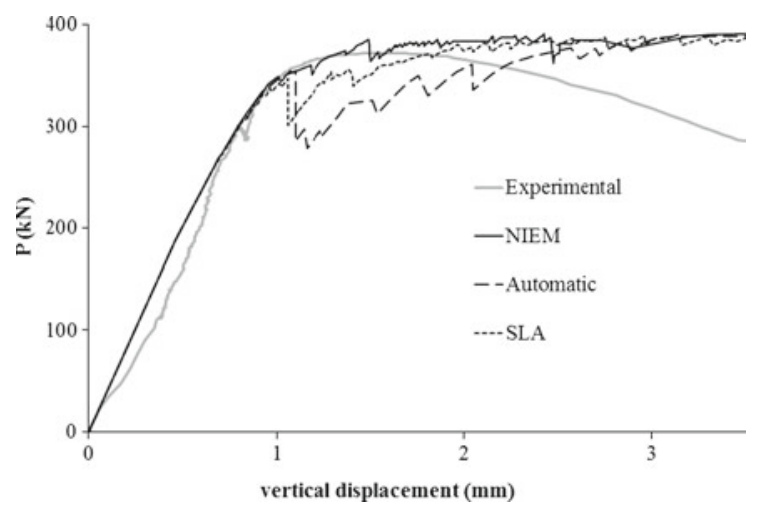

Fig. 20 Severes diagonal test: load-displacement curves for the panel without prescribed cracks

approach to reach a $3.5 \mathrm{~mm}$ displacement at the control point, 702 steps for the NIEM (65 total steps-9.3\%) and 614 (103 total steps-14.7\%) for the automatic method.

In this example, the result obtained with the automatic method presents the largest deviation from the experimental result. This is due to two main reasons: (i) a large number of total steps is used and (ii) conversely to the $S L A$, no regularisation of the material parameters is adopted in the total approach. The best approximation of the experimental result is obtained with the NIEM.

In some cases, a major crack developed across the height of the specimen. In order to evaluate the significance of this crack pattern, a second model was tested, with a prescribed crack defined according to the major crack identified in one of the tests (see Fig. 21). The use 
Fig. 21 Severes diagonal test: photographs at failure on experimental tests (courtesy of the Severes Project)
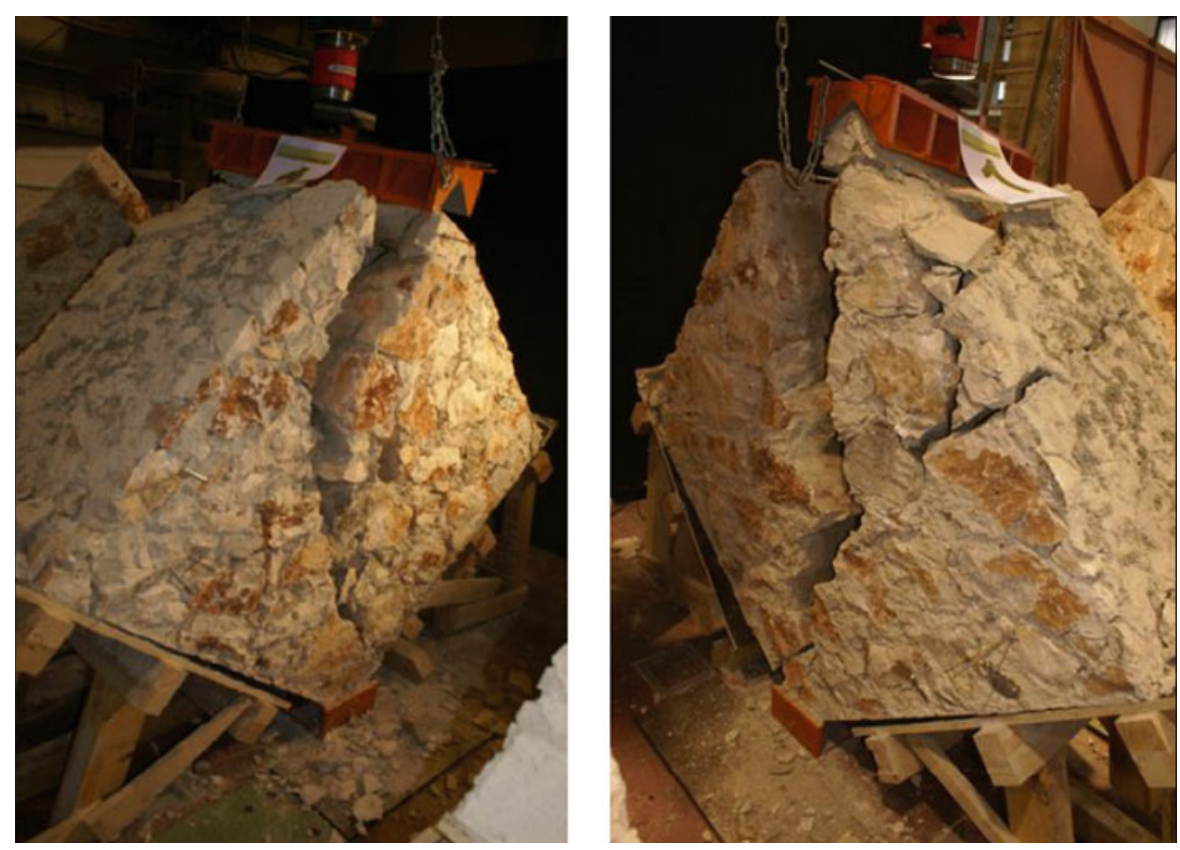

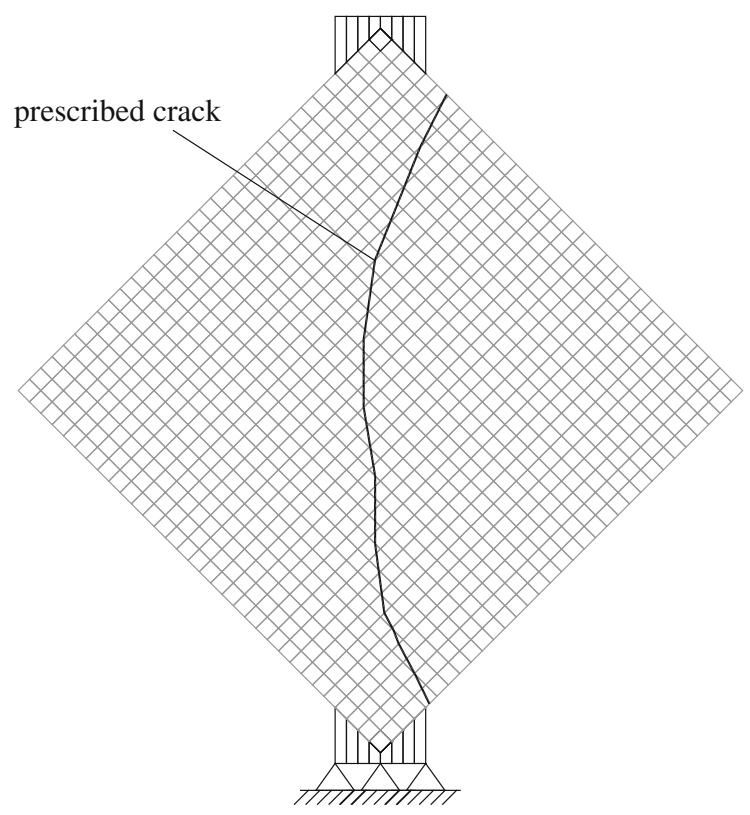

Fig. 22 Severes diagonal test: finite element mesh with prescribed crack

of a prescribed crack allows for a specific predefinition but new cracks can also form in the remaining elements of the bulk (see Fig. 22). In this second model, the load displacement curves do not show significant changes from the curves obtained with the first model since, apart from a perfect definition of the major crack, the

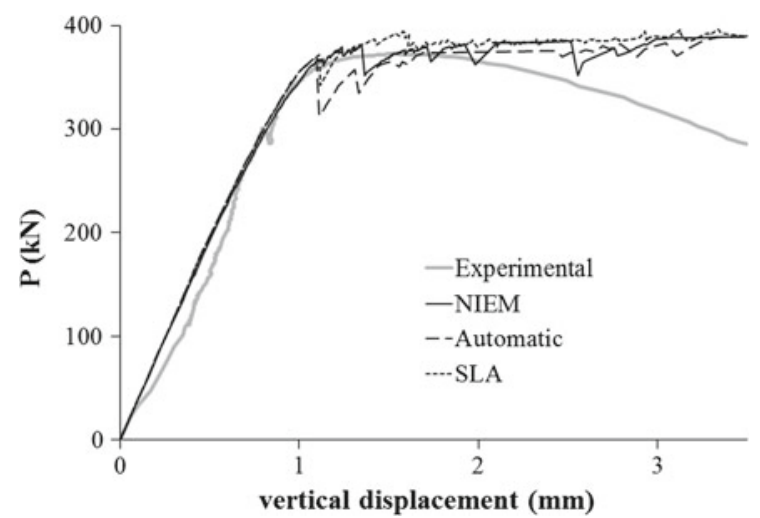

Fig. 23 Severes diagonal test: load-displacement curves for the panel with prescribed crack

remaining cracks are similar in both cases (Figs. 23, 24).

The three methods prove to overcome numerical difficulties and approximately depict the final deformed state of the two tested wall panels, built with hydraulic mortar.

\subsection{Prenotched concrete dam}

Another example for the proposed methods is the prenotched concrete dam, experimentally and numerically tested by Barpi and Valente (2000). The study is based 


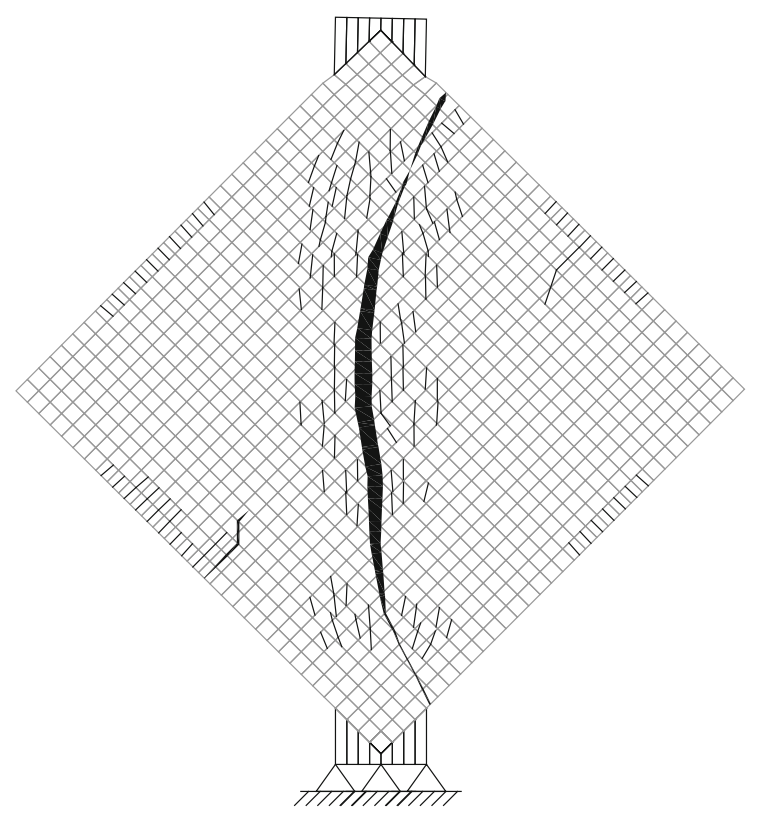

Fig. 24 Severes diagonal test: deformed finite element mesh and crack pattern using NIEM and a prescribed crack

on an experimental test of a dam, with the corresponding structural scheme depicted in Fig. 25.

The material parameters are adopted from Barpi and Valente (2000): Young's modulus $E=35,700 \mathrm{~N} / \mathrm{mm}^{2}$; Poisson's coefficient $v=0.1$; dead-weight $\rho=$ $2,400 \mathrm{~kg} / \mathrm{m} 3$; tensile strength $f_{t 0}=3.6 \mathrm{~N} / \mathrm{mm}^{2}$ and fracture energy $G_{F}=0.184 \mathrm{~N} / \mathrm{mm}$. Additionally, the constitutive law for the discontinuities is assumed to follow a multilinear law using 10 branches based on the exponential softening function where the shear
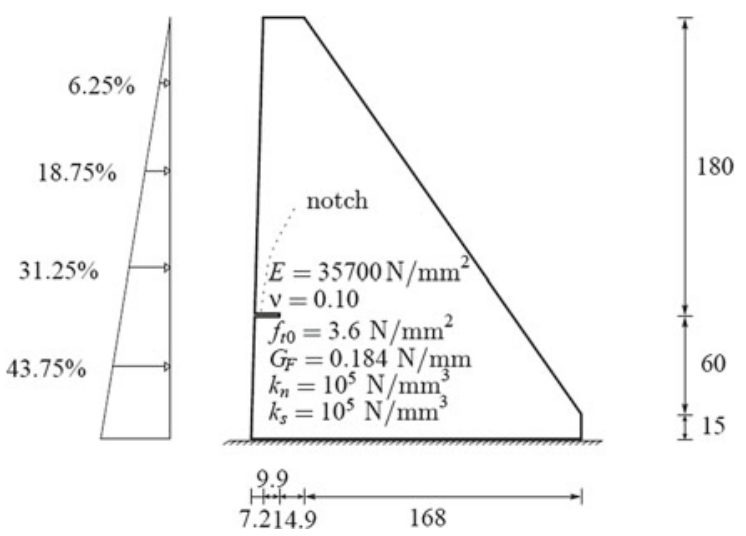

Fig. 25 Prenotched gravity dam model: structural scheme $(30 \mathrm{~cm}$ width, dimensions in $\mathrm{cm})$

stiffness gradually drops to zero (Dias-da-Costa et al. 2010).

The FE mesh is composed of 1848 bilinear elements as shown in Fig. 26.

Two load stages are applied to the structure: the dead load is first applied using an incremental approach since no bifurcation points will emerge; afterwards the water pressure in front of the dam is gradually increased. On the second loading case, the three methods are used by enforcing the monotonic increase of the relative CMOD. The load-CMOD curves are presented in Fig. 27. It is concluded that all numerically obtained results are similar. Since these formulations are not mixed-mode, differences can be found on the descending post-peak branch. This is why numerically, the load decays faster than experimentally. However
Fig. 26 Prenotched gravity dam model: FE mesh composed of 1,848 elements

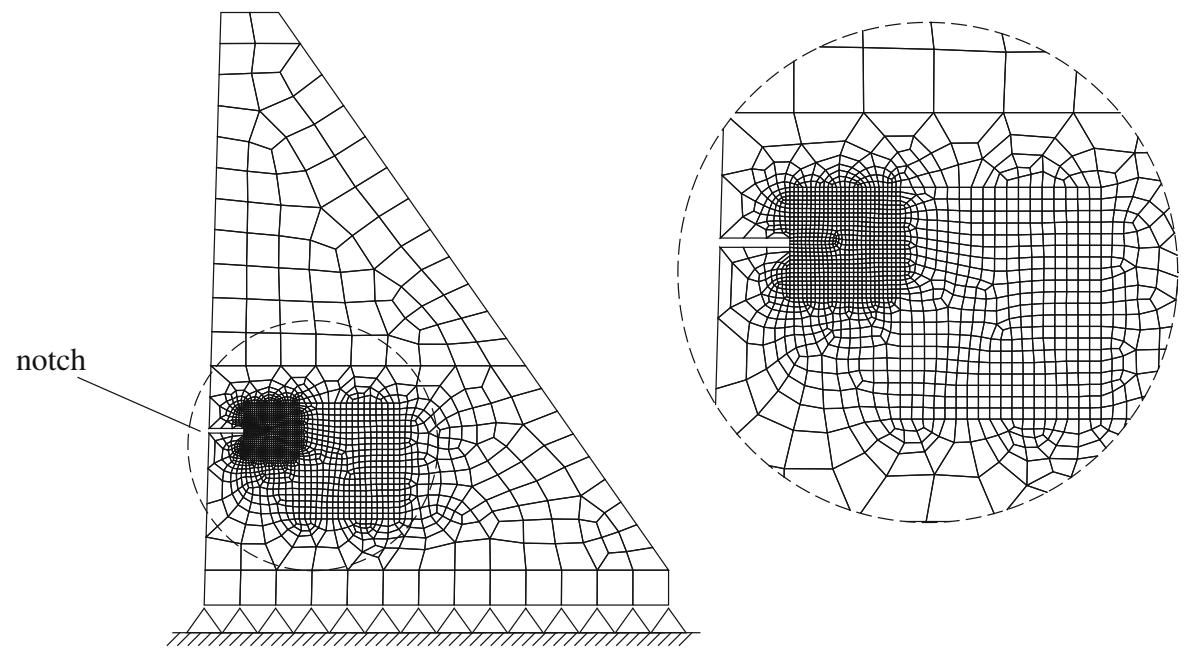




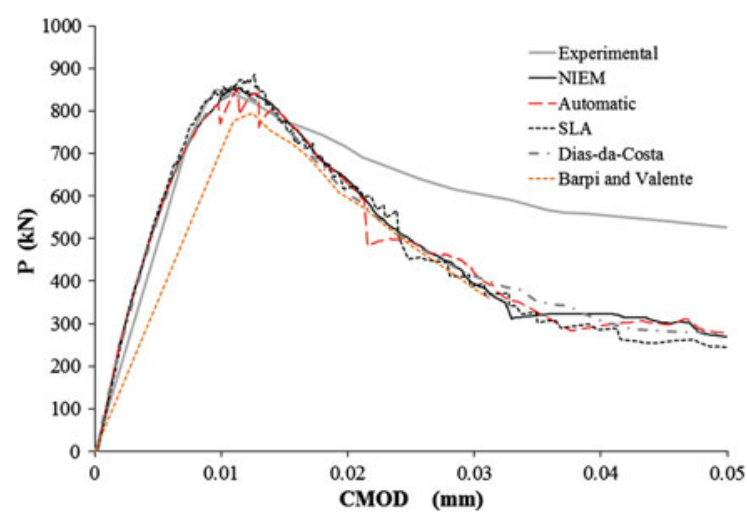

Fig. 27 Prenotched gravity dam model: load-CMOD curves

these results are similar to those obtained by Barpi and Valente (2000) and Dias-da-Costa et al. (2010).

The deformed mesh is equivalent on the three formulations, with a major crack developing from the notch with a slight downward inclination (Fig. 28).

The obtained elemental traction-jump envelopes clearly prove that the use of the NIEM leads to more consistent results, since the material's envelope is followed almost completely (compare Figs. 29, 30, 31). The SLA diagrams have the implicit tendency to concentrate teeth at the beginning of the envelope thus presenting increased accuracy for stresses near the peak load, whereas the opposite occurs for the lower secant stiffness zones. The use of the automatic method leads to better results than the SLA; however, on critical bifurcation points, the transition to the total approach causes significant deviations from the envelope, leading to reduced fracture energy consumption. The NIEM almost eliminates these deviations on total steps, because there is a much smoother transition due to the secant stiffness update. In this case, the faster method is the automatic method with 542 steps, followed by the SLA with 580 steps and by the NIEM with 720 steps. Regarding the accuracy of the results

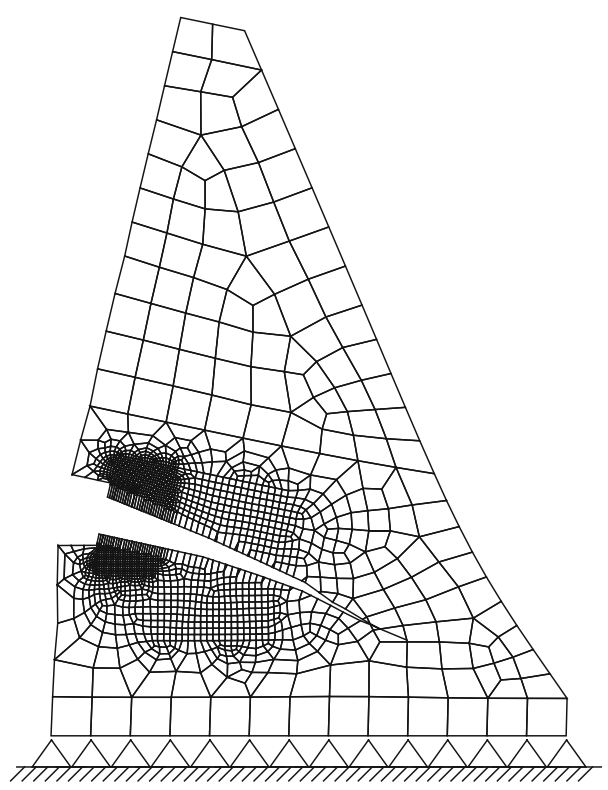

Fig. 28 Prenotched gravity dam model: deformed mesh when $\mathrm{CMOD}=0.35 \mathrm{~mm}$ (displacements amplified 500 times)

presented in Figs. 29, 30, 31, the mean value of fracture energy consumption was $+14.80 \%$ with the SLA, $-8.2 \%$ with the automatic method and $-1.4 \%$ with the NIEM. In this example, the number of transitions to the total approach is very small using both the automatic method ( 6 steps-1.11\%) and the NIEM (15-1.83\%).

In this model, the comparison between the optimised iterative solution and the non-iterative solutions is the following: the iterative solution took $198 \mathrm{~s}$ whereas the automatic solution took $128 \mathrm{~s}$, the NIEM took $171 \mathrm{~s}$ and the SLA took $155 \mathrm{~s}$.

\subsection{Reinforced concrete beam}

As an example of application to reinforced concrete, a test on a moderately reinforced concrete beam is pre-

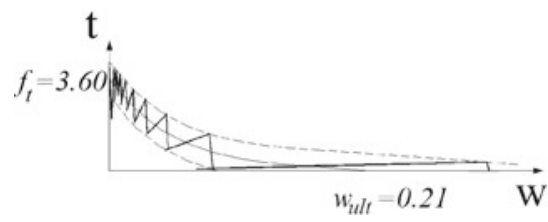

(a)

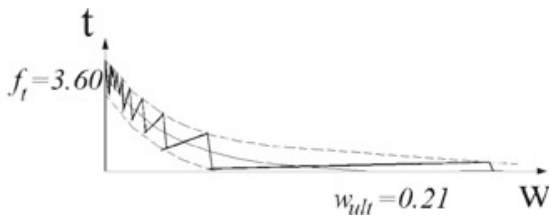

(b)

Fig. 29 Prenotched gravity dam: traction-jump envelope (MPa-mm) using the total approach. a Integration point at the most opened crack, $\mathbf{b}$ integration point at the middle of the full crack 
Fig. 30 Prenotched gravity dam: traction-jump envelope (MPa-mm), using the automatic method. a Integration point at the most opened crack, b integration point at the middle of the full crack

Fig. 31 Prenotched gravity dam: traction-jump envelope (MPa-mm), using the NIEM. a Integration point at the most opened crack, $\mathbf{b}$ integration point at the middle of the full crack

Fig. 32 Beam tested by Burns and Seiss (1962), Lowes (1999) (dimensions in $\mathrm{mm}$ )

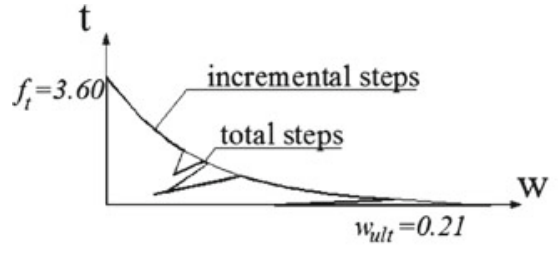

(a)

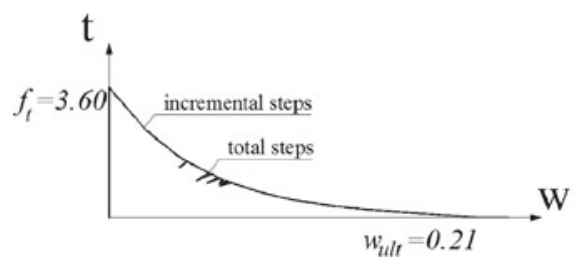

(a)

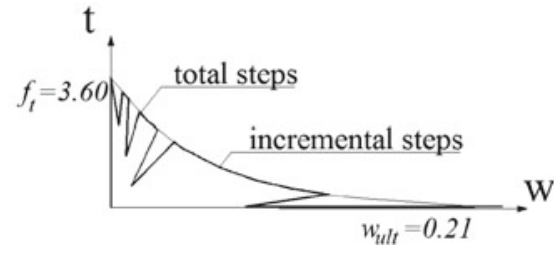

(b)

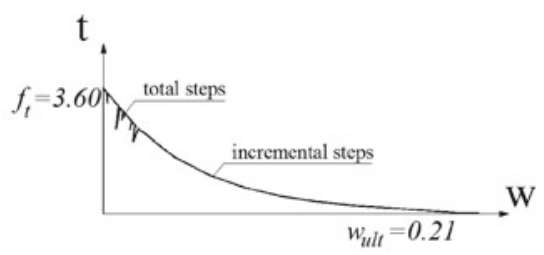

(b)
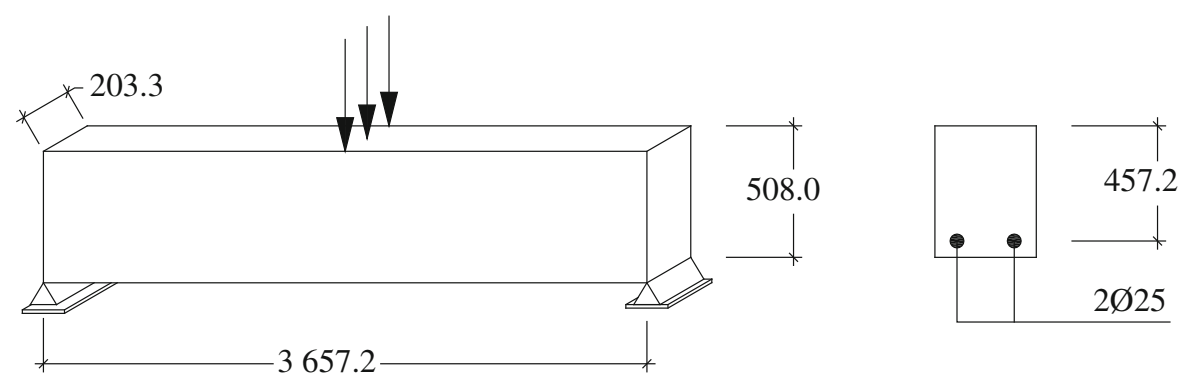

sented. This numerical test is compared to a numerical analysis presented by Lowes (1999), obtained with the Newton-Raphson method and the arc-length path following technique. In this test a smeared crack approach was adopted, in which the tensile steel reinforcement was bonded to concrete by means of interfaces under mode-II fracture.

This beam was experimentally also by Burns and Seiss (1962) whose results are a benchmark for several numerical models. This is a simply supported beam, loaded at mid-span (Fig. 32), with tensile reinforcement and no stirrups.

In this type of structures several nonlinearities occur, namely: (i) concrete softening under tensile stress, (ii) hardening of the steel reinforcement, (iii) bond-slip between concrete and steel; and (iv) concrete crushing under compression. First, generalised distributed cracking is found until localisation starts to occur, in which some cracks continue developing and others tend to close. This localisation is extremely difficult to capture with iterative methods, which often lead to nonconvergence.

The material characteristics used in the tests are presented on Table 1. It is noticed that the adopted value
Table 1 Burns and Seiss beam test: material properties

\begin{tabular}{lll}
\hline Material properties & Concrete & Steel \\
\hline Young's modulus (MPa) & 24,115 & 203,255 \\
Poisson's coefficient & 0.175 & 0.30 \\
Tensile strength $(\mathrm{MPa})$ & 2.41 & 309.36 \\
Compressive strength $(\mathrm{MPa})$ & 33.21 & 309.36 \\
Fracture energy (N/mm) & 0.26 & N/A \\
\hline
\end{tabular}

for the fracture energy is significantly higher than usual (Lowes 1999).

Symmetry conditions are enforced to decrease the problem size (Fig. 33). The finite element mesh is composed of 360 bilinear elements for concrete with elastoplastic behaviour under compression and embedded discontinuities with bilinear softening laws under tension (Alfaiate and Sluys 2002; Alfaiate et al. 2003). For the reinforcement, 36 truss elements are used superimposed on the concrete mesh using 36 mode-II zero thickness interface elements obeying the MC90 bondslip law (CEB 1991). The eventual development of 
Fig. 33 Burns and Seiss beam test: finite element mesh

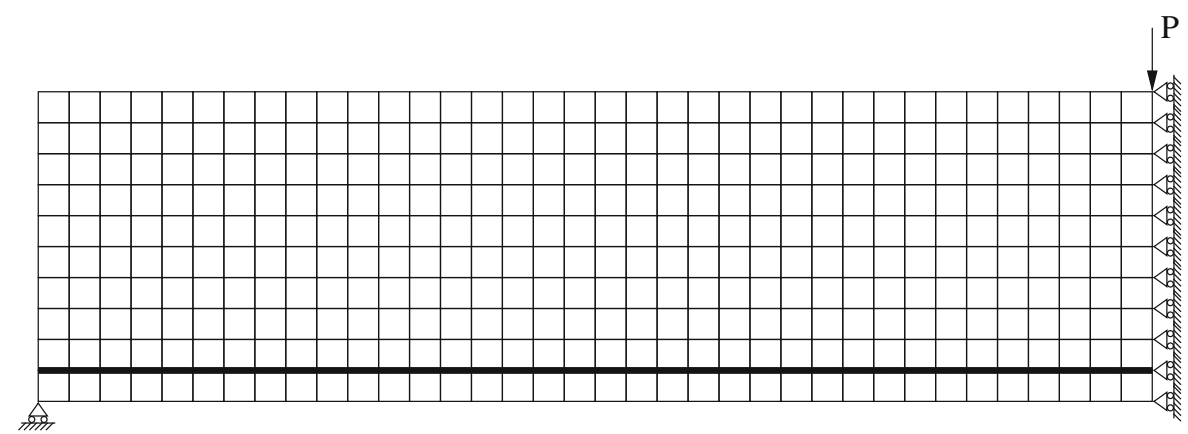

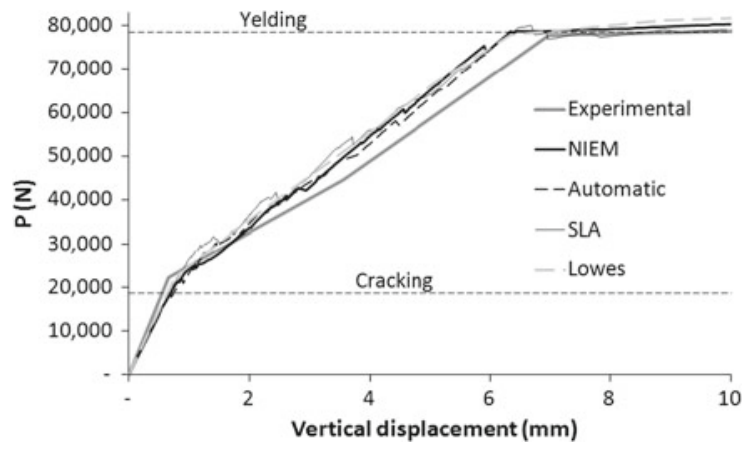

Fig. 34 Burns and Seiss beam test: load-displacement curves

cracking at mid-span is simulated with 10 interface elements at the symmetry line.

In Fig. 34, the load-displacement curves are presented. The difference to the experimental curve, also detected in Lowes (1999), seems to be a result of the inaccuracy of the material values, namely the excessive fracture energy.

In Fig. 35 the deformed mesh and embedded discontinuity crack pattern obtained with the NIEM are presented. Similar results were obtained for the other two used methods.

In this test yielding of the bond-slip between reinforcement and concrete was not detected; furthermore,
Table 2 Burns and Seiss beam: normal finite element mesh, parameter results using the three methods

\begin{tabular}{llll}
\hline Parameter & \multicolumn{3}{l}{ Method } \\
\cline { 2 - 4 } & SLA & Automatic & NIEM \\
\hline Steps $\left(\mathrm{N}^{\circ}\right)$ & 347 & 247 & 331 \\
Total steps $\left(\mathrm{N}^{\circ}\right)$ & 347 & 25 & 22 \\
Total steps $(\%)$ & 100 & 10 & 7 \\
Max load $(\mathrm{N})$ & 80,178 & 78,153 & 82,068 \\
Crack load $(\mathrm{N})$ & 25,736 & 21,369 & 23,887 \\
Yield load $(\mathrm{N})$ & 79,410 & 77,140 & 78,720 \\
Cracking $(\mathrm{mm})$ & 0.177 & 0.192 & 0.180 \\
Mean Gf $(\%)$ & 113.03 & 77.65 & 96.20 \\
\hline
\end{tabular}

the most compressed concrete element did not reach the compressive strength (Table 2).

Concrete initial cracking and yielding of the steel bars are depicted on the load-displacement chart. In terms of cracking, it is relevant to compare the obtained traction-jump history at the tip of the widest crack, with the theoretical material envelope (Figs. 36, 37, 38).

Using the SLA a larger deviation is obtained. In the automatic method a smaller underestimation of the fracture energy is obtained, caused by the transitions to the total approach. The NIEM provides an envelope
Fig. 35 Burns and Seiss beam test: deformed mesh and crack pattern using the NIEM for a mid-span displacement of $10 \mathrm{~mm}$ (displacements magnified 10 times, crack openings magnified 20 times)

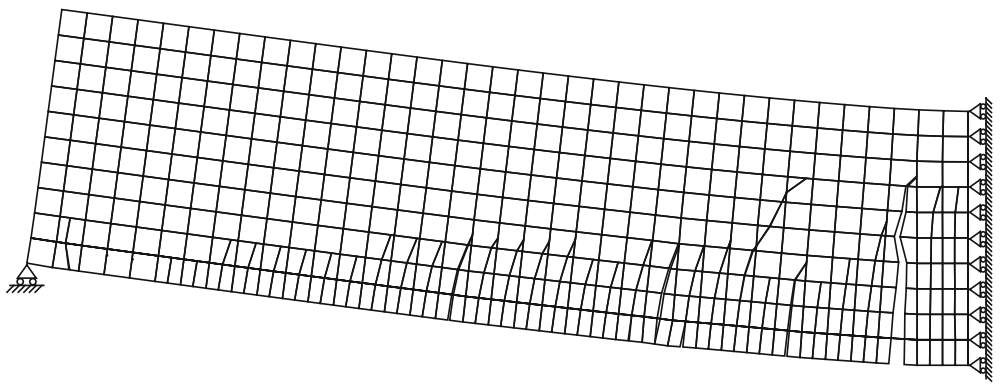




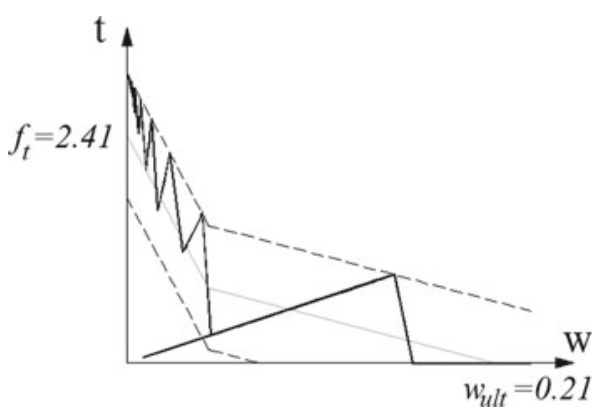

Fig. 36 Burns and Seiss beam test: normal finite element mesh, traction-jump envelope (MPa-mm) using the $S L A\left(G_{F}=\right.$ $0.2963 \mathrm{~N} / \mathrm{mm}: 113.9 \%$ )

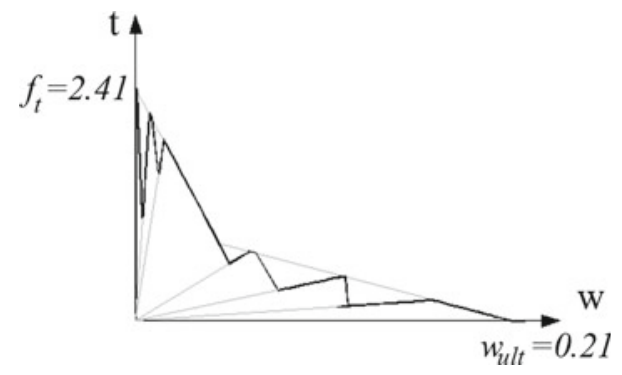

Fig. 37 Burns and Seiss beam test: traction-jump envelope (MPa-mm) using the automatic method $\left(G_{F}=0.2133 \mathrm{~N} / \mathrm{mm}-82.0 \%\right)$

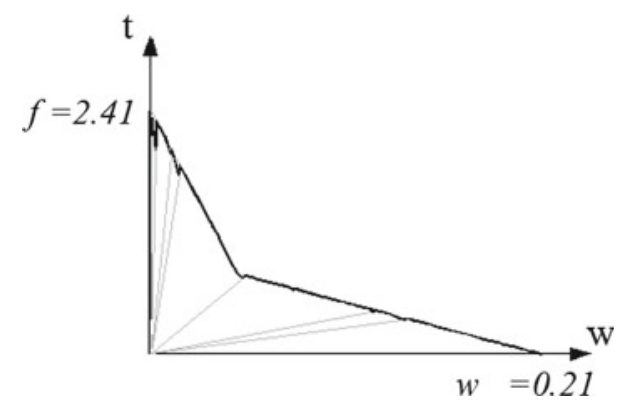

Fig. 38 Burns and Seiss beam test: traction-jump (MPa-mm) envelope using the $\operatorname{NIEM}\left(G_{F}=0.2555 \mathrm{~N} / \mathrm{mm}-98.3 \%\right)$

very close to the theoretical one, due to much smaller deviations from the correct material law.

\section{Conclusions}

Two innovative methods were presented which provide a powerful tool to the numerical simulation of complex nonlinear structures. These methods combine incremental and total approaches, thus taking advantage of the accuracy of incremental approaches with the effectiveness of total approaches, in order to overcome convergence problems at critical bifurcation points for incremental-iterative methods.

These formulations are non-iterative and do not require a fictitious adjustment of the constitutive law, since the use of total steps on the whole analysis is not significant to lead to an important underestimation of the fracture energy. In fact, the theoretical versus computed energy consumption ratio is adequate in one case and almost equal to one in the second case, with mean values of $83 \%$ for the automatic method and $99 \%$ for the NIEM.

The following main conclusions can be drawn from the three presented examples:

(i) the use of an energy consumption criterion seems an adequate solution control technique, allowing the use of incremental approaches on models with sharp snap-backs;

(ii) both the load-displacement curves and crack patterns obtained agree very well with experimental observation;

(iii) the NIEM shows a better approximation of the material laws when compared to the automatic method with an acceptable increase in computing time.

Finally it can be stated that the introduced formulations, although non-iterative, adequately follow an incremental approach and, as such, can be used with cyclic loading since material memory is kept during the analysis.

Acknowledgments This work is supported by FEDER funds through the Operational Programme for Competitiveness Factors-COMPETE - and by Portuguese funds through FCTPortuguese Foundation for Science and Technology under Project No. FCOMP-01-0124-FEDER-020275 (FCT ref. PTDC/ ECM/119214/2010).

\section{References}

Alfaiate J, Simone A, Sluys LJ (2003) Non-homogeneous displacement jumps in strong embedded discontinuities. Int J Solids Struct 40: 5799-5817. doi:10.1016/ S0020-7683(03)00372-X

Alfaiate J, Sluys LJ (2002) Analysis of a compression test on concrete using strong embedded discontinuities. In: Mang HA, Rammerstorfer FG, Eberhardsteiner J (eds) WCCM $\mathrm{V}$, fifth world congress on computational mechanics. Wien, Austria

Alfaiate J, Wells GN, Sluys LJ (2002) On the use of embedded discontinuity elements with crack path continuity for mode I 
and mixed mode fracture. Eng Fract Mech 69(6): 661-686. doi:10.1016/S0013-7944(01)00108-4

Barpi F, Valente S (2000) Numerical simulation of prenotched gravity dam models. J Eng Mech 126(6): 611-619. doi:10. 1061/(ASCE)0733-93399(2000)126:6(611)

Billington SL (2009) Nonlinear and sequentially linear analysis of tensile strain hardening cement-based composite beams in flexure. In: Hendriks M, Billington SL (eds) Computational modeling workshop on concrete, masonry and on fiber-reinforced composites, pp 7-10, Delf, The Netherlands

Burns NH, Seiss CP (1962) Load-deformation characteristics of beam-column connections in reinforced concrete. Report technical report, Engineering Studies SRS 234. Department of Civil Engineering, University of California, Berkeley

CEB (1991) CEB-FIP Model Code 1990. Thomas Telford, London

Crisfield MA (1984) Difficulties with current numerical models for reinforced-concrete and some tentative solutions. Comput Aided Anal Des Concr Struct (1):331-358

Dias-da-Costa D, Alfaiate J, Sluys LJ, Júlio E (2009) Towards a generalization of a discrete strong discontinuity approach. Comput Methods Appl Mech Eng 198(47-48): 3670-3681. doi:10.1016/j.cma.2009.07.013

Dias-da-Costa D, Alfaiate J, Sluys LJ, Júlio E (2010) A comparative study on the modelling of discontinuous fracture by means of enriched nodal and element techniques and interface elements. Int J Fract 161(1):97-119. doi:10.1007/ s10704-009-9432-6

Gago A, Milosevic J, Lopes M, Bento R (2011, submitted) Shear strength of rubble stone masonry walls. Bull Earthq Eng

Galvez JC, Elices M, Guinea GV, Planas J (1998) Mixed mode fracture of concrete under proportional and nonproportional loading. Int J Fract 94(3): 267-284. doi:10.1023/A: 1007578814070

Graça-e-Costa R (2005) Modelação de vigas de betão armado reforçadas com chapas metálicas. MSc thesis, Instituto Superior Técnico, Universidade Técnica de Lisboa, Portugal

Graça-e-Costa R, Alfaiate J (2006) The numerical analysis of reinforced concrete beams using embedded discontinuities. SDHM Struct Durab Health Monit 1: 11-17. doi:10.3970/ sdhm.2006.002.011

Gutiérrez MA (2004) Energy release control for numerical simulations of failure in quasi-brittle solids. Commun Numer Methods Eng 20(1): 19-29. doi:10.1002/cnm.649
Invernizzi S, Trovato D, Hendriks MAN, van de Graaf AV (2011) Sequentially linear modelling of local snap-back in extremely brittle structures. Eng Struct 33(5): 16171625. doi:10.1016/j.engstruct.2011.01.031

Lowes LN (1999) Finite element modeling of reinforced concrete beam-column bridge connections. PhD thesis, University of California, Berkeley

Milosevic J, Bento R, Gago A, Lopes M (2010) Seismic vulnerability of old masonry buildings-SEVERES project. Report 1. Instituto Superior Técnico, Lisbon. www.severes.org

Oliver J, Huespe AE, Cante JC (2008) An implicit/explicit integration scheme to increase computability of non-linear material and contact/friction problems. Comput Methods Appl Mech Eng 197(21-24): 1865-1889. doi:10.1016/j. cma.2007.11.027

Rots JG (2001) The role of structural modelling in preserving Amsterdam architectural city heritage. In: Lourenço PB, Roca P (eds) Historical constructions. Guimarães, Portugal, pp 685-696

Rots JG, Belletti B, Invernizzi S (2008) Robust modeling of RC structures with an "event-by-event" strategy. Eng Fract Mech 75(3-4): 590-614. doi:10.1016/j.engfracmech.2007. 03.027

Schlangen E (1993) Experimental and numerical analysis of fracture process in concrete. PhD thesis, Delft University of Technology, The Netherlands

Slobbe AT, Hendriks MAN, Rots JG (2012) Sequentially linear analysis of shear critical reinforced concrete beams without shear reinforcement. Finite Elem Anal Des 50(0): 108-124. doi:10.1016/j.finel.2011.09.002

Verhoosel CV., Remmers JJC, Gutiérrez MA (2009) A dissipation-based arc-length method for robust simulation of brittle and ductile failure. Int J Numer Methods Eng 77(9): 12901321. doi:10.1002/nme. 2447

Xing H, Zhang J (2009) Finite element modelling of non-linear deformation of rate-dependent materials using a R-minimum strategy. Acta Geotechnica 4(2): 139-148. doi:10. 1007/s11440-009-0090-7

Yamada Y, Yoshimura N, Sakurai T (1968) Plastic stress-strain matrix and its application for the solution of elastic-plastic problems by the finite element method. Int J Mech Sci 10(5): 343-354. doi:10.1016/0020-7403(68)90001-5 